\title{
Ionized Hypomagnesemia Is Associated With Increased Incidence of Postoperative Atrial Fibrillation After Esophageal Resection: A Retrospective Study
}

\author{
Kotaro Hizuka $^{1}$, Takao Kato ${ }^{1}$, Yuki Shiko ${ }^{2,1}$, Yohei Kawasaki ${ }^{3,}$,, 1 , Kaoru Koyama ${ }^{1}$ \\ 1. Anesthesiology, Saitama Medical Center, Saitama Medical University, Kawagoe, JPN 2. Biostatistics, Clinical \\ Research Center, Chiba University Hospital, Chiba, JPN 3. Emergency Medicine, Japanese Red Cross College of Nursing, \\ Tokyo, JPN
}

Corresponding author: Kotaro Hizuka,sms_rugby@yahoo.co.jp

\begin{abstract}
Introduction: Postoperative atrial fibrillation (POAF) is common after surgery for esophageal cancer and may prolong hospitalization and elevate mortality. POAF and hypomagnesemia are linked, but this is based on studies showing an association of POAF with serum total magnesium (tMg). In contrast, the relationship of POAF with ionized magnesium (iMg), which has physiological activity, has not been examined. In this study, the association between hypomagnesemia and POAF was investigated retrospectively to examine iMg as a possible predictive factor for POAF.
\end{abstract}

Methods: The subjects were 151 patients who underwent right transthoracoabdominal subtotal esophagectomy at Saitama Medical Center between January 2011 and December 2020. The incidence of POAF and predictive factors were examined retrospectively. Perioperative predictive factors were subjected to univariate analysis, and items with $\mathrm{P}<0.1$ were then included in multivariate analysis, along with five potential POAF predictors reported in the literature (age, gender, body mass index, hypertension, and diabetes mellitus). $\mathrm{P}<0.05$ was regarded as significant in the multivariate analysis.

Results: Of the 151 patients, 34 (23\%) developed POAF. In univariate analysis, six factors with $\mathrm{P}<0.1$ (oral statin, dyslipidemia, iMg level after anesthesia induction, maximum and minimum iMg during surgery, and iMg level immediately before admission to ICU) were identified. In multivariate analysis including these and the five literature factors as explanatory variables, iMg immediately before admission to ICU emerged as a predictive factor for POAF (iMg $\geqslant 0.46 \mathrm{mmol} / \mathrm{L}$, OR 0.32 , 95\%CI 0.14-0.74, $\mathrm{p}=0.01$ ) (standard iMg range: 0.48 $0.60 \mathrm{mmol} / \mathrm{L})$.

Conclusion: The iMg level immediately before admission to ICU may be associated with the development of POAF. A further study is needed to evaluate changes in iMg in the ICU and iMg at the time of onset of POAF.

Review began $07 / 26 / 2021$ Review ended 08/11/2021 Published 08/11/2021

\section{() Copyright 2021}

Hizuka et al. This is an open access article distributed under the terms of the Creative Commons Attribution License CC-BY 4.0., which permits unrestricted use, distribution, and reproduction in any medium, provided the original author and source are credited.
Categories: Anesthesiology, Cardiac/Thoracic/Vascular Surgery, Cardiology

Keywords: postoperative atrial fibrillation, arrhythmia, thoracic surgery, esophageal cancer, electrolyte disturbance, ionized magnesium

\section{Introduction}

Postoperative atrial fibrillation (POAF) occurs at a high rate and markedly increases the risk of cerebral and myocardial infarction, and associated mortality $[1,2]$. The causes of POAF include acute factors directly associated with surgery (inflammation and noradrenaline activation) and chronic or progressive factors, such as remodeling of the heart (left atrial enlargement), but the detailed pathophysiological mechanism has yet to be determined [3].

The incidence of POAF in surgeries other than cardiac or thoracic surgery is about 3\% [4], whereas that after surgery for esophageal cancer is $16 \%$, and this high rate is associated with an increased risk of postoperative complications, in addition to higher mortality [5]. The high incidence of POAF after surgery for esophageal cancer is due to the common patient background, which often includes cigarette smoking and alcohol intake, and to the physical stress of the operation on the heart, preoperative changes in atrial substrate, and noradrenaline stimulation due to surgical inflammation, all of which are thought to have effects because the esophagus is located close to the left atrium $[3,6]$.

Studies of POAF after surgery for esophageal cancer have identified several preoperative predictive factors, including age, gender, body mass index (BMI), and a history of hypertension, diabetes mellitus, heart disease, or lung disease $[2,3,5,7-9]$. None of these studies investigated intraoperative findings as predictive factors, but an association of intraoperative hypomagnesemia with POAF has been reported [10]. The 
Magnesium (Mg) level is generally measured as the serum total magnesium (tMg), but only ionized magnesium (iMg) has physiological activity and changes in iMg are inconsistent with changes in $\mathrm{tMg}$ [9]. The intraoperative iMg level has been found to be a risk factor for POAF after coronary artery bypass surgery using an artificial heart-lung machine [10].

In this study, we hypothesized that perioperative iMg is related to POAF after surgery for esophageal cancer. To examine this hypothesis, perioperative iMg was examined in patients undergoing right transthoracoabdominal surgery for esophageal cancer, and potential predictive factors for POAF were evaluated in an analysis of perioperative findings and previously identified patient-based risk factors.

\section{Materials And Methods}

A retrospective observational study was performed after approval by Saitama Medical Center Ethics Committee (approval number 2555-II). The STROBE checklist was utilized. The subjects were 185 patients who received curative surgery for esophageal cancer at Saitama Medical Center between January 1, 2011 and December 31, 2020, and were retrospectively followed for at least three months after surgery until March 31, 2021. Data were obtained from electronic medical records (EMRs). Patients who underwent elective right transthoracoabdominal subtotal esophagectomy were included in the study. Patients with atrial fibrillation (AF) induced by curative surgery for esophageal cancer using a different surgical procedure and those in whom AF was noted before surgery were excluded. Patients with no postoperative records available were also excluded from the study.

Surgery was performed under combined epidural and general anesthesia. General anesthesia was maintained by inhalation of sevoflurane or desflurane and total intravenous anesthesia with continuous administration of propofol, as selected by the physician in charge. For pain control, epidural anesthesia and remifentanil were concomitantly used during surgery and epidural anesthesia was used postoperatively. For differential lung thoracotomy, a double-lumen tube or bronchial blocker was used. After surgery, the patient was admitted to the ICU with intubation. Extubation was performed on the following day when there were no problems with consciousness, respiration, and circulation, and the patient was then managed in a general ward for two days after surgery.

The definition of POAF in this study was AF (including paroxysmal AF) that was newly developed within seven days after surgery. Continuous monitoring was used postoperatively in the ICU. After the patient returned to a general ward, arrhythmia was detected by a nurse during a routine vital signs check or a check for a symptomatic condition. Age, gender, BMI, history of hypertension or diabetes mellitus, and intraoperative iMg were obtained from EMRs.

Age, gender, height $(\mathrm{cm})$, body weight $(\mathrm{kg})$, BMI, resting heart rate/mean arterial pressure, the presence or absence of underlying disease (diabetes mellitus, dyslipidemia, hypertension, history of ischemic heart disease, cardiovascular disease, peripheral arterial disease), medications, cardiac ultrasonography findings (left atrial diameter, left ventricular ejection fraction), blood test results (hematocrit, potassium, creatinine, eGFR, ICGR15), ASA-PS classification, cancer stage, and the presence or absence of preoperative chemotherapy were examined as preoperative patient background factors. Operation time, fluid infusion/blood transfusion volume, blood loss, urine output, total balance, administered drugs, intraoperative body temperature, and perioperative blood test data (intraoperative iMg, potassium, blood glucose levels) were surveyed as perioperative factors. The presence or absence of POAF, hospitalization days and three months mortality were examined as postoperative outcomes. Intraoperative iMg was measured using a blood gas analyzer (Stat pHOx UltraTM, Nova Biomedical, Waltham, MA, USA). The standard value of iMg was set at $0.48-0.60 \mathrm{mmol} / \mathrm{L} \mathrm{[11].}$

Summary statistics (mean \pm standard deviation) were calculated for continuous variables, and the number and rate of cases were calculated for categorical variables. To identify factors influencing the development of POAF, the subjects were divided into those with and without POAF. Univariate analysis of continuous variables was performed by Student t-test, and that of categorical variables was performed by Chi-squared test. Variables with $\mathrm{P}<0.1$ in univariate analysis and five factors (age, gender, BMI, hypertension, and diabetes) associated with POAF in previous studies were used as explanatory variables in subsequent analyses. The cut-off value of each continuous variable was determined and the variable was binarized. Sensitivity and specificity were calculated using a ROC curve, and the Youden index was used as a reference. Multivariate logistic regression analysis using the stepwise method (threshold of $\mathrm{P}<0.1$ ) was performed using the binarized factors as explanatory variables and the presence or absence of POAF as the response variable. When performing the stepwise method, the five variables (age, gender, BMI, hypertension, and diabetes mellitus) were fixed to remain in the analysis, and then the variable selection was performed for the other factors. A two-sided significance level of $5 \%$ was used in all statistical analyses. All calculations were performed in SAS ver. 9.4 (SAS Institute, Cary, NC, USA).

\section{Results}

Curative surgery for esophageal cancer was performed as an elective operation in 185 patients in total between January 2011 and December 2020, of whom 34 were excluded from the study. Of the remaining 151 


\section{Cureus}

patients, 34 developed POAF, giving an incidence of $23 \%$ (Figure 1). The POAF onset day and number of cases are shown in Figure 2. POAF most commonly developed two days after surgery in many cases (15 cases, $44 \%)$.

Eligible participants $(\mathrm{n}=185)$

Jan 2011 - Dec 2020

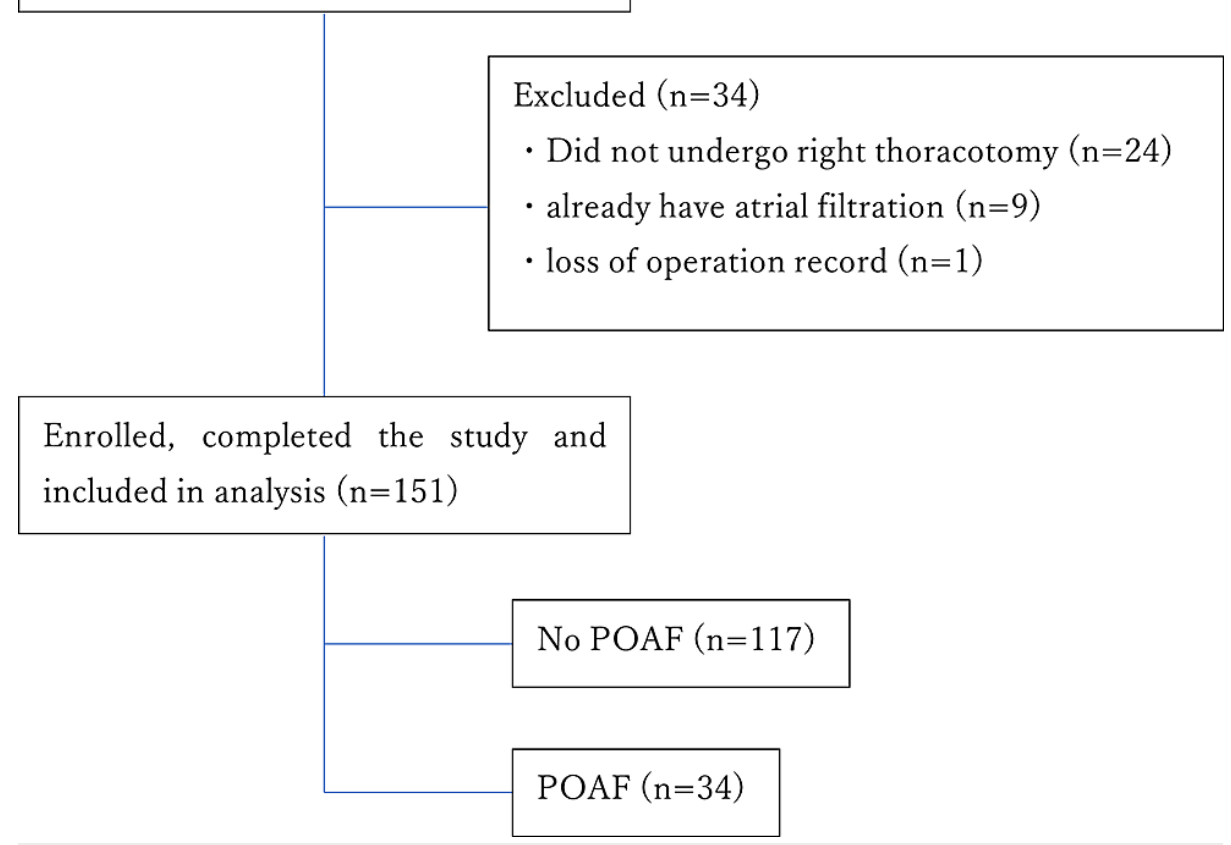

FIGURE 1: Flowchart for identifying patients between January 2011 and December 2020 at Saitama Medical Center, Saitama Medical University, Kawagoe, Japan

POAF: postoperative atrial fibrillation

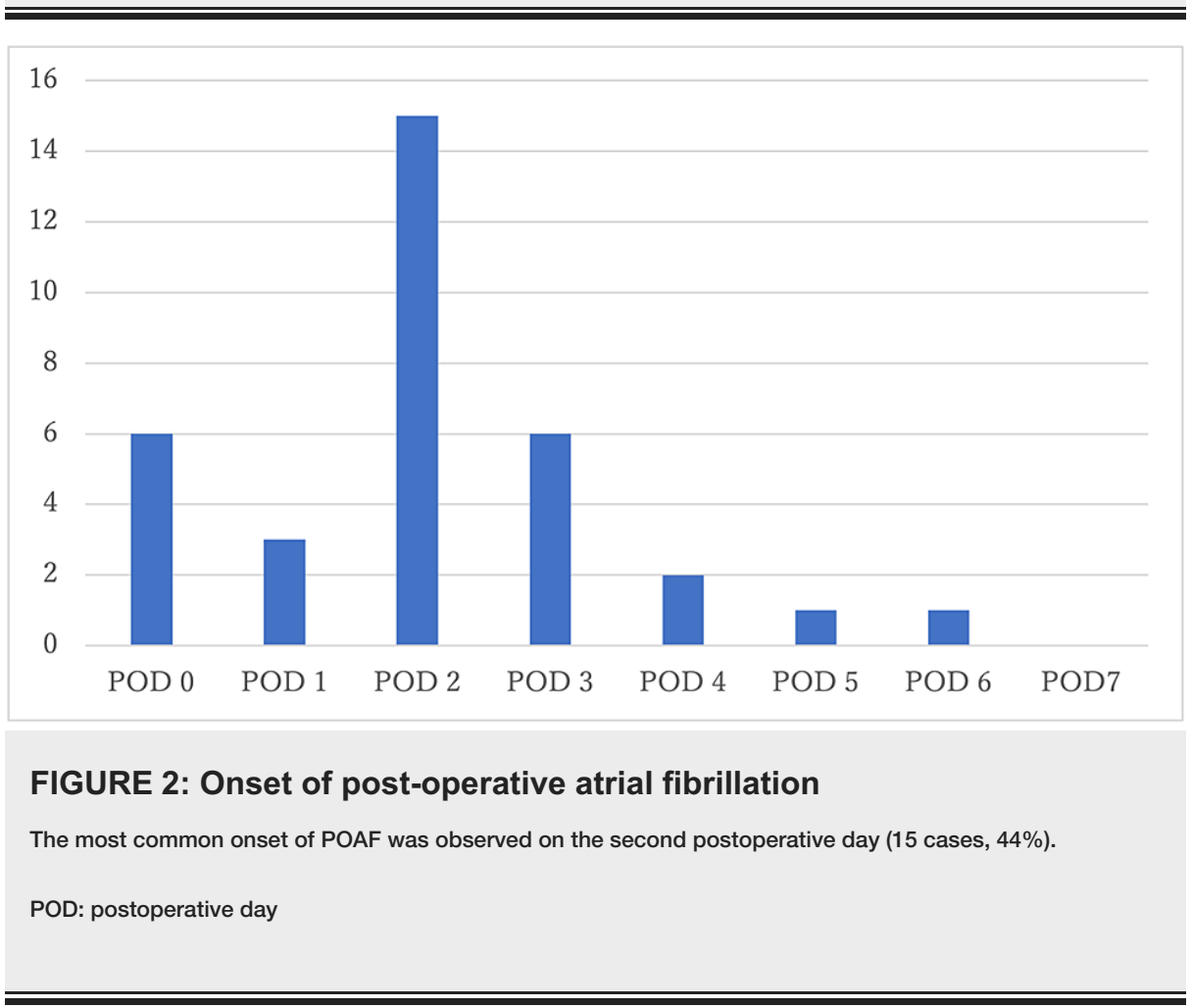


The results of univariate analysis are shown in Table 1 for preoperative factors and patient background, Table 2 for intraoperative factors, and Table 3 for postoperative factors. Table 1 shows oral statin administration and dyslipidemia were associated with a lower risk of POAF in preoperative factors. Table 2 shows iMg (before surgery, immediately before admission to ICU, maximum and minimum) were associated with a higher risk of POAF in intra-operative factors. Table 3 shows there were no significant differences in postoperative factors, including length of hospital stay and three months mortality. Due to the lack of iMg records in the ICU, we were unable to obtain data on postoperative iMg levels or on iMg levels at the onset of POAF. Oral statin administration, dyslipidemia, iMg level after anesthesia induction, intraoperative maximum and minimum iMg levels, and iMg level immediately before admission to ICU all had a value of $\mathrm{P}<0.1$ in univariate analysis. Among these variables and the known factors, continuous variables were binary transformed for multivariate logistic regression analysis, and the respective cut-off values were calculated as follows, iMg immediately before admission to ICU: $0.46 \mathrm{mmol} / \mathrm{L}$, iMg after induction of general anesthesia: $0.51 \mathrm{mmol} / \mathrm{L}$, intra-operative Maximum iMg: $0.51 \mathrm{mmol} / \mathrm{L}$, Intra-operative Minimum iMg: $0.46 \mathrm{mmol} / \mathrm{L}$, Age: 72 years, BMI: $23 \mathrm{~kg} / \mathrm{m}^{2}$ (Table 4). In this multivariate analysis, iMg immediately before admission to ICU emerged as an independent variable associated with POAF ( $\geqslant 0.46 \mathrm{mmol} / \mathrm{L}, \mathrm{OR}: 0.32,95 \% \mathrm{CI}: 0.14-0.74$, $\mathrm{p}=0.01$ ), in addition to the five previously reported factors (Table 5). Among the known factors, gender, hypertension, and diabetes mellitus did not show significant differences.

\begin{tabular}{|c|c|c|c|c|}
\hline & $\begin{array}{l}\text { All patient } \\
\text { ( } n=151)\end{array}$ & $\begin{array}{l}\text { No POAF } \\
(n=117)\end{array}$ & $\begin{array}{l}\text { POAF } \\
(n=34)\end{array}$ & value \\
\hline Age (years) & $67.94 \pm 36.87$ & $67.69 \pm 8.48$ & $68.71 \pm 7.75$ & 0.49 \\
\hline Gender (male) & $131(87 \%)$ & $101(86 \%)$ & $30(88 \%)$ & 0.77 \\
\hline Height (cm) & $162.83 \pm 7.61$ & $162.48 \pm / .81$ & $164.03 \pm 6.89$ & 0.30 \\
\hline Bodyweight (kg) & $57.23 \pm 9.70$ & $57.56 \pm 9.57$ & $56.09 \pm 10.22$ & 0.44 \\
\hline Body mass index $\left(\mathrm{kg} / \mathrm{m}^{2}\right)$ & $21.57 \pm 3.32$ & $21.79 \pm 3.22$ & $20.82 \pm 3.60$ & 0.14 \\
\hline Resting heart rate (bpm) & $73.44 \pm 13.93$ & $74.03 \pm 14.60$ & $71.41 \pm 11.31$ & 0.34 \\
\hline Resting mean arterial pressure (mmHg) & $92.70 \pm 13.18$ & $92.15 \pm 13.22$ & $94.56 \pm 13.05$ & 0.35 \\
\hline ICG-R15(\%) & $9.28 \pm 4.41$ & $9.38 \pm 4.91$ & $9.14 \pm 4.19$ & 0.80 \\
\hline HbA1c (\%) & $5.99 \pm 0.83$ & $6.01 \pm 0.91$ & $5.88 \pm 0.67$ & 0.52 \\
\hline Hematocrit (\%) & $37.52 \pm 4.83$ & $37.38 \pm 4.89$ & $38.01 \pm 4.65$ & 0.50 \\
\hline Potassium (mEq/L) & $4.39 \pm 0.418$ & $4.39 \pm .041$ & $4.41 \pm 0.44$ & 0.75 \\
\hline Cr (mg/dL) & $0.84 \pm 0.26$ & $0.85 \pm 0.29$ & $0.80 \pm 0.14$ & 0.34 \\
\hline eGFR* $\left(\mathrm{ml} / \mathrm{min} / 1.73 \mathrm{~m}^{2}\right)$ & $72.33 \pm 19.20$ & $72.09 \pm 20.78$ & $73.14 \pm 12.56$ & 0.78 \\
\hline Left ventricular ejection fraction (\%) & $69.70 \pm 7.07$ & $69.95 \pm 7.15$ & $69.09 \pm 7.89$ & 0.55 \\
\hline Left atrial diameter (mm) & $33.66 \pm 5.55$ & $33.58 \pm 5.88$ & $34.12 \pm 5.55$ & 0.64 \\
\hline \multicolumn{5}{|l|}{ Comorbidity } \\
\hline Diabetes mellitus & $16(11 \%)$ & $15(13 \%)$ & $1(3 \%)$ & 0.10 \\
\hline Dyslipidemia & $19(13 \%)$ & $18(15 \%)$ & $1(2.9 \%)$ & 0.05 \\
\hline Hypertension & $73(48 \%)$ & $60(51 \%)$ & $13(38 \%)$ & 0.18 \\
\hline Ischemic heart disease & $6(4 \%)$ & $6(5.1 \%)$ & $0(0 \%)$ & 0.18 \\
\hline Cerebrovascular disease & $12(8 \%)$ & $9(7.7 \%)$ & $3(8.8 \%)$ & 0.83 \\
\hline Peripheral arterial disease & $4(3 \%)$ & $2(1.7 \%)$ & $2(5.9 \%)$ & 0.18 \\
\hline \multicolumn{5}{|l|}{ Medications } \\
\hline Calcium blocker & $53(35 \%)$ & $43(37 \%)$ & $10(29 \%)$ & 0.43 \\
\hline $\begin{array}{l}\text { Angiotensın II receptor blocker or angiotensın-convertıng enzyme } \\
\text { inhibitors }\end{array}$ & $39(26 \%)$ & $30(26 \%)$ & $9(26 \%)$ & 0.92 \\
\hline beta-blocker & $6(4 \%)$ & $5(4.3 \%)$ & $1(2.9 \%)$ & 0.73 \\
\hline
\end{tabular}




\section{Cureus}

\begin{tabular}{|c|c|c|c|c|}
\hline alpha1-blocker & $5(3 \%)$ & $5(4.2 \%)$ & $0(0 \%)$ & 0.22 \\
\hline Statin & $14(9 \%)$ & $14(12 \%)$ & $0(0 \%)$ & 0.03 \\
\hline Aspirin & $9(6 \%)$ & $6(5.1 \%)$ & $3(8.8 \%)$ & 0.42 \\
\hline Anticoagulant & $10(7 \%)$ & $7(6.0 \%)$ & $3(8.8 \%)$ & 0.56 \\
\hline ASA-PS classification 1 & 29 (19\%) & 22 (19\%) & $7(20 \%)$ & \multirow{3}{*}{0.54} \\
\hline ASA-PS classification 2 & $118(78 \%)$ & $91(78 \%)$ & 27 (79\%) & \\
\hline ASA-PS classification 3 & $4(3 \%)$ & $4(3 \%)$ & $0(0 \%)$ & \\
\hline Cancer stage (0) & $2(1 \%)$ & $1(1 \%)$ & $1(3 \%)$ & \multirow{5}{*}{0.68} \\
\hline Cancer stage (I) & $15(11 \%)$ & 10(9\%) & $5(15 \%)$ & \\
\hline Cancer stage (II) & 45 (30\%) & $36(31 \%)$ & $9(26 \%)$ & \\
\hline Cancer stage (III) & 77 (51\%) & $60(51 \%)$ & $17(50 \%)$ & \\
\hline Cancer stage (IV) & $12(8 \%)$ & $10(8.6 \%)$ & $2(6 \%)$ & \\
\hline Preoperative chemotherapy & $45(30 \%)$ & $38(32 \%)$ & $7(21 \%)$ & 0.18 \\
\hline
\end{tabular}

TABLE 1: Patient background (preoperative factors) and results of univariate analysis between no POAF and POAF patient

Continuous data are presented as mean \pm standard deviation and categorical data as number (percentage).

POAF: post-operative atrial fibrillation; ICG-R15: Indocyanine green retention test after 15 minutes; ASA-PS: American Society of Anesthesiologists Physical Status

*eGFR was calculated by the equation devised by the Japanese Society of Nephrology [12]. 


\section{Cureus}

\begin{tabular}{|c|c|c|c|c|}
\hline & All patient $(n=151)$ & No POAF $(n=117)$ & POAF (n=34) & P-value \\
\hline Potassium before surgery $(\mathrm{mmol} / \mathrm{L})$ & $4.0 \pm 0.44$ & $4.0 \pm 0.47$ & $4.0 \pm 0.36$ & 0.86 \\
\hline iMg before surgery $(\mathrm{mmol} / \mathrm{L})$ & $0.52 \pm 0.07$ & $0.53 \pm 0.07$ & $0.49 \pm 0.07$ & 0.0074 \\
\hline iMg immediately before admission to ICU ( $\mathrm{mmol} / \mathrm{L})$ & $0.49 \pm 0.08$ & $0.51 \pm 0.08$ & $0.45 \pm 0.07$ & 0.0008 \\
\hline Maximum iMg (mmol/L) & $0.54 \pm 0.08$ & $0.55 \pm 0.09$ & $0.51 \pm 0.06$ & 0.0094 \\
\hline Minimum iMg (mmol/L) & $0.47 \pm 0.07$ & $0.48 \pm 0.08$ & $0.44 \pm 0.07$ & 0.023 \\
\hline Maxımum-minımum iMg ( $(\mathrm{mmol} / \mathrm{L})$ & $0.07 \pm 0.06$ & $0.07 \pm 0.06$ & $0.06 \pm 0.04$ & 0.46 \\
\hline Blood glucose before surgery (mg/dl) & $129 \pm 31$ & $129 \pm 31$ & $130 \pm 32$ & 0.87 \\
\hline Maximum blood glucose (mg/dL) & $189 \pm 40$ & $189 \pm 43$ & $188 \pm 31$ & 0.94 \\
\hline Minimum body temperature $\left({ }^{\circ} \mathrm{C}\right)$ & $35 \pm 0.8$ & $35 \pm 0.8$ & $35 \pm 0.7$ & 0.98 \\
\hline Body temperature after surgery $\left({ }^{\circ} \mathrm{C}\right)$ & $36 \pm 0.9$ & $35 \pm 0.9$ & $35 \pm 0.9$ & 0.66 \\
\hline Magnesium sulfate & $5(3.3 \%)$ & $5(4.3 \%)$ & $0(\%)$ & 0.22 \\
\hline Insulin & $18(12 \%)$ & $14(12 \%)$ & $4(12 \%)$ & 0.97 \\
\hline Noradrenaline & $80(52 \%)$ & $66(56 \%)$ & $14(41 \%)$ & 0.12 \\
\hline Dopamine & $11(7.3 \%)$ & $10(8.6 \%)$ & $1(2.9 \%)$ & 0.27 \\
\hline Dobutamine & $4(2.6 \%)$ & $2(1.7 \%)$ & $2(5.9 \%)$ & 0.18 \\
\hline Operation time (min) & $393 \pm 78$ & $391 \pm 75$ & $403 \pm 87$ & 0.43 \\
\hline Total fluid infusion (mL) & $3,950 \pm 1,197$ & $3,963 \pm 1,183$ & $3,904 \pm 1258$ & 0.80 \\
\hline Blood transfusion (red blood cell, mL) & $177 \pm 368$ & $175 \pm 379$ & $181 \pm 330$ & 0.93 \\
\hline Blood transfusion (fresh frozen plasma, $\mathrm{mL}$ ) & $78 \pm 223$ & $78 \pm 220$ & $78 \pm 234$ & 0.99 \\
\hline Blood loss (mL) & $680 \pm 587$ & $663 \pm 524$ & $736 \pm 771$ & 0.52 \\
\hline Urine output (mL) & $1,063 \pm 700$ & $1,107 \pm 733$ & $910 \pm 557$ & 0.15 \\
\hline Total balance $(\mathrm{mL})$ & $2,462 \pm 1,019$ & $2,447 \pm 994$ & $2,517 \pm 1,115$ & 0.73 \\
\hline
\end{tabular}

TABLE 2: Intraoperative factors and results of univariate analysis between no POAF and POAF patients

Continuous data are presented as mean \pm standard deviation and categorical data as number (percentage).

POAF: post-operative atrial fibrillation; iMg: ionized magnesium 


\section{Cureus}

\begin{tabular}{|c|c|c|c|c|}
\hline & All patient $(n=151)$ & No POAF (n=117) & POAF $(n=34)$ & P-value \\
\hline Noradrenaline & $40(26 \%)$ & $30(26 \%)$ & $10(29 \%)$ & 0.66 \\
\hline C-reactive protein (mg/dL) & $13 \pm 5.5$ & $13 \pm 5.3$ & $13 \pm 6.1$ & 0.79 \\
\hline Creatinine kinase (U/L) & $823 \pm 375$ & $803 \pm 402$ & $909 \pm 456$ & 0.23 \\
\hline Acute kidney injury* & $4(2.6 \%)$ & $2(1.7 \%)$ & 2 (5.9\%) & 0.18 \\
\hline Extubation (POD) & $1.2 \pm 1.1$ & $1.1 \pm 1.1$ & $1.4 \pm 1.1$ & 0.24 \\
\hline Hospitalization days & $45 \pm 37$ & $56 \pm 54$ & $42 \pm 31$ & 0.15 \\
\hline Three months mortality & $2(1.3 \%)$ & $1(0.9 \%)$ & $1(2.9 \%)$ & 0.35 \\
\hline
\end{tabular}

\section{TABLE 3: Postoperative factors and results of univariate analysis between no POAF and POAF}

patients

Continuous data are presented as mean \pm standard deviation and categorical data as number (percentage).

POAF: post-operative atrial fibrillation; POD: postoperative day

*The Kidney Disease Improving Global Outcomes (KDIGO) classification was used for the diagnosis of acute kidney injury.

\begin{tabular}{|c|c|c|c|c|c|c|}
\hline & & \multicolumn{2}{|l|}{ No POAF } & \multicolumn{2}{|l|}{ POAF } & \multirow{2}{*}{ P-value } \\
\hline & & Number & $\%$ & Number & $\%$ & \\
\hline \multirow{2}{*}{ iMg immediately before admission to ICU（mmol/L） } & $<0.46$ & 28 & 24 & 18 & 53 & \multirow{2}{*}{0.0012} \\
\hline & $\geq 0.46$ & 89 & 76 & 16 & 47 & \\
\hline \multirow{2}{*}{ iMg after induction of general anesthesia ( $\mathrm{mmol} / \mathrm{L})$} & $<0.51$ & 40 & 34 & 21 & 62 & \multirow{2}{*}{0.0039} \\
\hline & $\geq 0.51$ & 77 & 66 & 13 & 38 & \\
\hline \multirow{2}{*}{ Intra-operative maximum iMg (mmol/L) } & $<0.51$ & 34 & 29 & 19 & 56 & \multirow{2}{*}{0.0039} \\
\hline & $\geq 0.51$ & 83 & 71 & 15 & 44 & \\
\hline \multirow{2}{*}{ Intra-operative minimum iMg (mmol/L) } & $<0.46$ & 44 & 38 & 19 & 56 & \multirow{2}{*}{0.057} \\
\hline & $\geq 0.46$ & 73 & 62 & 15 & 44 & \\
\hline \multirow{2}{*}{ Age (years) } & $<72$ & 76 & 65 & 16 & 47 & \multirow{2}{*}{0.060} \\
\hline & $\geq 72$ & 41 & 35 & 18 & 53 & \\
\hline \multirow[t]{2}{*}{ Body mass index $\left(\mathrm{kg} / \mathrm{m}^{2}\right)$} & $<23$ & 72 & 62 & 28 & 82 & \multirow[t]{2}{*}{0.024} \\
\hline & $\geq 23$ & 45 & 38 & 6 & 18 & \\
\hline \multicolumn{7}{|l|}{ Data are presented as numbers (percentage). } \\
\hline POAF: postoperative atrial fibrillation; iMg: ionized magnesi & re unit & & & & & \\
\hline
\end{tabular}




\section{Cureus}

\begin{tabular}{|llllll|}
\hline Variables & & Odds Ratio & $95 \%$ Confidence Interval & P-value \\
\hline Age (years) & $\geq 72$ & 2.86 & 1.19 & 6.86 & 0.02 \\
Gender & Male & 1.77 & 0.48 & 6.51 & 0.39 \\
Body mass index $\left(\mathrm{kg} / \mathrm{m}^{2}\right)$ & $\geq 23$ & 0.28 & 0.10 & 0.80 & 0.02 \\
Hypertension & No morbidity & 1.80 & 0.74 & 4.35 & 0.19 \\
Diabetes mellitus & No morbidity & 5.00 & 0.56 & 44.36 & 0.15 \\
iMg immediately before admission to ICU (mmol/L) & $\geq 0.46$ & 0.32 & 0.14 & 0.74 & 0.01 \\
\hline
\end{tabular}

TABLE 5: Predictors of postoperative atrial fibrillation by multivariate analysis

iMg: ionized magnesium; ICU: intensive care unit

\section{Discussion}

In this study, $23 \%$ of patients developed POAF after elective right transthoracoabdominal subtotal esophagectomy. In multivariate analysis, iMg immediately before admission to ICU was identified as an independent POAF-predictive factor. POAF most frequently developed two days after surgery and $34 \%$ of all cases occurred within two days after surgery.

Hypomagnesemia (tMg $<1.5 \mathrm{mg} / \mathrm{dl}$ or $<0.62 \mathrm{mmol} / \mathrm{L}$ ) is common in patients with advanced cancer and in those admitted to an ICU $[13,14]$. Cisplatin used in preoperative chemotherapy for esophageal cancer induces hypomagnesemia [15], and thus, patients undergoing surgery for esophageal cancer are more likely to have hypomagnesemia compared with other cases. The action potential of cardiac muscle cells is transmitted through voltage-dependent sodium, potassium, and calcium ion channels, and changes in the function of these channels may lead to arrhythmia. Mg acts as a membrane-stabilizer by blocking calcium channels and adjusting cell membrane sodium-potassium transport and also inhibits catecholamine release from the adrenal gland and adrenergic nerve ending [16]. These properties result in an anti-arrhythmic effect of Mg. Similar to esophageal cancer surgery, POAF is common after coronary artery bypass surgery, in which an association of hypomagnesemia and prevention of POAF by magnesium preparations have been shown [17-19]. However, tMg was examined in these studies and iMg was rarely measured, despite its association with physiological activity [20]. Also, changes in iMg are inconsistent with changes in $\mathrm{tMg}$, and tMg may be within the normal range despite the intracellular magnesium content being low [9,21].

The current study showed the value of measuring iMg as a direct predictor of POAF. The cut-off value of iMg, $0.46 \mathrm{mmol} / \mathrm{L}$, was consistent with the range of clinical hypo-ionized hypomagnesemia [11], suggesting an indication for magnesium sulfate supplementation. Magnesium supplementation targeting $\mathrm{iMg} \geqslant 0.50$ $\mathrm{mmol} / \mathrm{L}$ during artificial heart-lung management has been shown to decrease the frequency of ventricular tachycardia and prolong the duration of sinus rhythm in coronary artery bypass surgery [22]. Setting a target value of iMg, which can be easily measured at the bedside, has been found to facilitate favorable management in the treatment of Torsades de pointes accompanying drug-induced long QT syndrome [23]. Magnesium supplementation has also been suggested to be useful for the prevention of POAF after surgery for esophageal cancer and cardiac surgery [6,24,25], and thus, perioperative iMg monitoring and $\mathrm{Mg}$ supplementation may prevent POAF.

The basis of the development of POAF after surgery for esophageal cancer is not completely clear, but the mechanism is multifactorial and various predictive factors have been investigated. In this study, the multivariate analysis included five previously established risk factors (age, gender, BMI, hypertension, and diabetes mellitus). In the 2014 AATS Guidelines for Prevention and Management of Perioperative Atrial Fibrillation and Flutter for Thoracic Surgical Procedures [6], age, gender, BMI, and hypertension are included as risk factors. Among these predictive factors, the most consistent is advanced age, and aging-associated atrial tissue remodeling is thought to increase the risk of POAF. Hypertension and diabetes mellitus can also alter atrial tissue [26]. Perioperative statin administration has been reported to decrease the incidence of POAF [27], and in the current study, the incidence of POAF was significantly lower in patients taking oral statins for hyperlipidemia in univariate analysis.

POAF developed after surgery for esophageal cancer in 34 (23\%) of the 151 patients in the current study. The most frequent onset time was two days after surgery, which occurred in $44 \%$ of cases, and POAF developed three days after surgery in $88 \%$ of the affected cases. In previous studies of esophageal cancer surgery, the incidence of POAF was $\geqslant 15 \%$ and the findings were consistent with our observation of peak development two days after surgery $[4,6]$. Few studies have examined the association of iMg with physiological activity 
and POAF development [20] and this is the first report to examine the relationship of iMg with POAF development after surgery for esophageal cancer.

This study has several limitations, including its retrospective design. A larger number of cases could not be included because medical records were not kept for more than 10 years, and this reduced our ability to adjust for confounding factors. As a result, of the factors identified in previous studies, there was no significant difference in gender, hypertension, or diabetes mellitus between POAF and non-POAF cases. Also, outcomes, such as mortality, could not be investigated because the number of cases was small and the incidence was low. In addition, $\mathrm{tMg}$ was not measured, which prevented examination of the correlation of $\mathrm{tMg}$ with POAF. There was no record of Mg measurement in the postoperative period, including in the ICU, so we were unable to investigate the relationship between iMg at the time of onset of POAF and that during POAF.

\section{Conclusions}

Predictive factors for POAF after surgery for esophageal cancer were investigated in a single-center retrospective study. In multivariate analysis, iMg immediately before admission to ICU was identified as a risk factor for POAF (iMg $\geqslant 0.46 \mathrm{mmol} / \mathrm{L}, \mathrm{OR}: 0.32,95 \% \mathrm{CI}: 0.14-0.74, \mathrm{p}=0.01$ ). This is the first study to show a possible association between ionized magnesium and POAF after esophageal resection. Further prospective studies on the relationship between ionized magnesium and POAF (and furthermore, the relationship with mortality) are needed.

\section{Additional Information \\ Disclosures}

Human subjects: Consent was obtained or waived by all participants in this study. Ethical Committee of Saitama Medical Center, Saitama Medical University issued approval 2555-II. Animal subjects: All authors have confirmed that this study did not involve animal subjects or tissue. Conflicts of interest: In compliance with the ICMJE uniform disclosure form, all authors declare the following: Payment/services info: All authors have declared that no financial support was received from any organization for the submitted work. Financial relationships: All authors have declared that they have no financial relationships at present or within the previous three years with any organizations that might have an interest in the submitted work. Other relationships: All authors have declared that there are no other relationships or activities that could appear to have influenced the submitted work.

\section{Acknowledgements}

We would like to thank PALABRA (www.palabra.co.jp) for English language editing.

\section{References}

1. AlTurki A, Marafi M, Proietti R, et al.: Major adverse cardiovascular events associated with postoperative atrial fibrillation after noncardiac surgery: a systematic review and meta-analysis. Circ Arrhythm Electrophysiol. 2020, 13:e007437. 10.1161/CIRCEP.119.007437

2. Oesterle A, Weber B, Tung R, et al.: Preventing postoperative atrial fibrillation after noncardiac surgery: a meta-analysis. Am J Med. 2018, 131:795-804. 10.1016/j.amjmed.2018.01.032

3. Maesen B, Nijs J, Maessen J, Allessie M, Schotten U: Post-operative atrial fibrillation: a maze of mechanisms. Europace. 2012, 14:159-74. 10.1093/europace/eur208

4. Sellers D, Srinivas C, Djaiani G: Cardiovascular complications after non-cardiac surgery . Anaesthesia. 2018, 73 Suppl 1:34-42. 10.1111/anae.14138

5. Schizas D, Kosmopoulos M, Giannopoulos S, et al.: Meta-analysis of risk factors and complications associated with atrial fibrillation after oesophagectomy. Br J Surg. 2019, 106:534-47. 10.1002/bjs.11128

6. Frendl G, Sodickson AC, Chung MK, et al.: 2014 AATS guidelines for the prevention and management of perioperative atrial fibrillation and flutter for thoracic surgical procedures. J Thorac Cardiovasc Surg. 2014, 148:e153-93. 10.1016/j.jtcvs.2014.06.036

7. Ma JY, Wang Y, Zhao YF, Wu Z, Liu LX, Kou YL, Yang JJ: Atrial fibrillation after surgery for esophageal carcinoma: clinical and prognostic significance. World J Gastroenterol. 2006, 12:449-52. 10.3748/wjg.v12.i3.449

8. Wells CI, Robertson JP, Campbell S, Al-Herz F, Rhind B, Young M: Impact of atrial fibrillation on long-term survival following oesophagectomy: a 21-year observational study. ANZ J Surg. 2018, 88:E268-72. 10.1111/ans. 14054

9. Reinhart RA: Magnesium metabolism. A review with special reference to the relationship between intracellular content and serum levels. Arch Intern Med. 1988, 148:2415-20. 10.1001/archinte. 148.11.2415

10. Baker WL: Treating arrhythmias with adjunctive magnesium: identifying future research directions . Eur Heart J Cardiovasc Pharmacother. 2017, 3:108-17. 10.1093/ehjcvp/pvw028

11. Nakayama T, Umehara K, Shirozu K, Sumie M, Karashima Y, Higashi M, Yamaura K: Association between ionized magnesium and postoperative shivering. J Anesth. 2021, 35:412-9. 10.1007/s00540-021-02914-y

12. Matsuo S, Imai E, Horio M, et al.: Revised equations for estimated GFR from serum creatinine in Japan . Am I Kidney Dis. 2009, 53:982-92. 10.1053/j.ajkd.2008.12.034

13. Deheinzelin D, Negri EM, Tucci MR, et al.: Hypomagnesemia in critically ill cancer patients: a prospective study of predictive factors. Braz J Med Biol Res. 2000, 33:1443-8. 10.1590/s0100-879x2000001200007 
14. Hansen BA, Bruserud Ø: Hypomagnesemia in critically ill patients. J Intensive Care. 2018, 6:21. 10.1186/s40560-018-0291-y

15. Lajer H, Daugaard G: Cisplatin and hypomagnesemia. Cancer Treat Rev. 1999, 25:47-58. 10.1053/ctrv.1999.0097

16. Rebollar RE, Palacios MVG, Guerrero JM, Torres LM: Magnesium sulfate in pediatric anesthesia: the super adjuvant. Paediatr Anaesth. 2017, 27:480-9. 10.1111/pan.13129

17. Abdel-Massih TE, Sarkis A, Sleilaty G, et al.: Myocardial extraction of intracellular magnesium and atrial fibrillation after coronary surgery. Int J Cardiol. 2012, 160:114-8. 10.1016/j.ijcard.2011.04.015

18. Reinhart RA, Marx JJ Jr, Broste SK, et al.: Myocardial magnesium: relation to laboratory and clinical variables in patients undergoing cardiac surgery. J Am College Cardiol. 1991, 17:651-6. 10.1016/s07351097(10)80179-2

19. Khan AM, Lubitz SA, Sullivan LM, et al.: Low serum magnesium and the development of atrial fibrillation in the community: the Framingham Heart Study. Circulation. 2013, 127:33-8. 10.1161/CIRCULATIONAHA.111.082511

20. Asano M, Kato K, Kawasaki Y, et al.: Risk factors for postoperative atrial fibrillation after coronary artery bypass grafting (in Japanese with English abstract). Cardiovasc Anesth. 2020, 24:41-7. 10.11478/jscva.20193-016

21. Yeh DD, Chokengarmwong N, Chang Y, et al.: Total and ionized magnesium testing in a surgical intensive care unit-opportunities for improved laboratory and pharmacy utilization. J Crit Care. 2017, 42:147-51. 10.1016/j.jcrc.2017.07.026

22. Wilkes NJ, Mallett SV, Peachey T, Di Salvo C, Walesby R: Correction of ionized plasma magnesium during cardiopulmonary bypass reduces the risk of postoperative cardiac arrhythmia. Anesth Analg. 2002, 95:82834, table of contents. 10.1097/00000539-200210000-00008

23. Matsuura C, Kato T, Koyama K: Successful management of refractory torsades de pointes due to druginduced long QT syndrome guided by point-of-care monitoring of ionized magnesium. Cureus. 2021, 13:e13939. 10.7759/cureus.13939

24. Arsenault KA, Yusuf AM, Crystal E, Healey JS, Morillo CA, Nair GM, Whitlock RP: Interventions for preventing post-operative atrial fibrillation in patients undergoing heart surgery. Cochrane Database Syst Rev. 2013, CD003611. 10.1002/14651858.CD003611.pub3

25. Fairley JL, Zhang L, Glassford NJ, Bellomo R: Magnesium status and magnesium therapy in cardiac surgery: a systematic review and meta-analysis focusing on arrhythmia prevention. J Crit Care. 2017, 42:69-77. 10.1016/j.jcrc.2017.05.038

26. Echahidi N, Pibarot P, O'Hara G, Mathieu P: Mechanisms, prevention, and treatment of atrial fibrillation after cardiac surgery. J Am Coll Cardiol. 2008, 51:793-801. 10.1016/j.jacc.2007.10.043

27. Zhen-Han L, Rui S, Dan C, Xiao-Li Z, Qing-Chen W, Bo F: Perioperative statin administration with decreased risk of postoperative atrial fibrillation, but not acute kidney injury or myocardial infarction: a meta-analysis. Sci Rep. 2017, 7:10091. 10.1038/s41598-017-10600-x 